\title{
Effect of Western Style Diet on NLRP3 Inflammasome and its Correlation with Metabolic Syndromes
}

\author{
Fatma Farag Abdel Hamid ${ }^{1}$, Dawoud Fakhary Habib ${ }^{2}$, \\ Aly Mohamed Ezz El-Arab ${ }^{3}$, Nadia A. Mohamed ${ }^{* 2}$, Rehab A. Mohamed ${ }^{4}$ \\ ${ }^{1}$ Biochemistry Department, Faculty of Science, Ain Shams University, Egypt \\ ${ }^{2}$ Medical Biochemistry Department, National Research Centre, Egypt \\ ${ }^{3}$ NutritionDepartment of Nutrition and Food Science, NRC, Cairo-Egypt \\ ${ }^{4}$ B.Sc. in Biochemistry 2011
}

\begin{abstract}
This work was done to study the effect of $(H F)$ and (HFF) on level of plasma glucose, insulin, kidney profile, lipid profile and antioxidant status compared to $(L F)$. The statistical analysis of the results showed that total cholesterol, triacylglycerol, plasma glucose, serum insulin, serum NLRP3, kidney nitric oxide and MDA were significantly increased in HFF compared to LF group. But PON1, GSH and HDL-C were significantly decreased in HFF compared to LF group. Our study shows a positive correlation between total cholesterol and triacylglycerol withNLRP3. Also there is a negative correlation between HDL-C, PONI and GSH withNLRP3. The conclusion that the inflammasome NLRP3 is an important marker that correlate between obesity, type 2 diabetes, oxidative stress and inflammation, So it is recommended to depend on as metabolic syndrome marker.
\end{abstract}

Keywords: WSD, diabetes, obesity, NLRP3, GSH, PON1, NO, MDA

\section{INTRODUCTION}

The development of agriculture, animal domestication and technological progress significantly lowered the cost and availability of food supply, creating a unique situation in the history of human evolution when caloric intake exceeded energy expenditure. Today, reduced physical activity and increased consumption of calorie-dense food are recognized as the main risk factors responsible for the epidemic growing of obesity in the modern society [1]. A better comparison of different dietary patterns is allowed at similar caloric intake, e.g. (1) The Mediterranean diet of southern Italian and Greek inhabitants is characterized by high contents of plant foods, fresh fruits, fish, and poultry, dairy products, and olive oil as the main source of fat. Red meat, saturated fat, and trans-fatty acids are low. The high content of monounsaturated fatty acids (MUFAs) such as oleic acid and the antioxidant and anti-inflammatory effects of olive oil may be responsible at least in part for the observed lower risk of coronary heart disease[2]. The adherence to a Mediterranean diet was found to exert protective effects against type 2 diabetes cardiovascular disease, and Parkinson's and Alzheimer's diseases with a lower risk of cancer [3]. (2) The Western Style Diet (WSD), called the meat-sweet diet or standard American diet, is characterized by an over availability of food, with high intakes of high-fat foods, high-sugar desserts and drinks as well as high intakes of red meat, refined grains, and high-fat dairy products. WSD underlies the epidemic growing of obesity in the United States and throughout the world [4]. It has been recognized as the major contributor to metabolic disturbances, hypertension, and cardiovascular diseases [5]. Also, it has been associated with an increased incidence of chronic kidney disease CKD [6]. A combination of dietary factors contributes to the impairment of renal vascularization, steatosis and inflammation, hypertension, and impaired renal hormonal regulation. It is thought to contribute to the increasing incidence of several chronic illnesses including cancer (especially colon and liver), non-alcoholic fatty liver disease (NAFLD)/non-alcoholic steatohepatitis (NASH) [7] and osteoporosis [8].Behind these similarities, however, high fat (HF) and high fat high fructose (HFF) diets affect whole-body lipid metabolism in different ways. In fact, during HF feeding an increased lipid supply to peripheral organs, and particularly to the liver, arises mainly from dietary lipids, while during HFF feeding an increased hepatic de novo lipogenesis is the main source of 
circulating lipid. Metabolic syndrome is defined as the presence of any three of the following five traits: abdominal obesity, hypertriacylglycerolmia, low high-density lipoprotein cholesterol, insulin resistance, and increased blood pressure. In rodents, fructose feeding leads to onset of symptoms of the metabolic syndrome, including hyperglycemia, hyperinsulinemia as well as obesity, hypertension, and increased levels of triacylglycerols, fatty acids and cholesterol [9]. In humans, consumption of a fructose-rich diet causes insulin resistance, increases uric acid, and stimulates de novo lipogenesis, resulting in increased levels of triacylglycerols, cholesterol, and small proatherogenic low-density lipoprotein particles [10]. Diabetes Mellitus (DM) is affecting a greater proportion of the world's population each year such that WHO predicts that DM will be the seventh leading cause of death by the year 2030 [11] as it essentially affects all systems of the body. Type 2 diabetes encompasses individuals who have insulin resistance. Most, but not all, patients with type 2 diabetes are obese[12]. Generalized insulin resistance occurs primarily as a result of obesity, a consequence of caloric excess, physical inactivity, genetics, and age. The nucleotide binding domain and leucine rich repeat protein3(NLRP3) inflammasome is a multiprotein, large cytoplasmic complex (>700 kDa), composed of a specific member of the NOD-like receptor protein (NLRP) subfamily, the adaptor protein named apoptosis-associated speck-like protein containing a CARD (ASC), and procaspase-1, which are preferentially expressed in adipose tissue macrophages (ATMs)[13]. Metabolic syndrome is characterized by a state of systemic low grade chronic inflammation [14]. However, the role of NLRP3 on the development of metabolic syndrome associated renal pathology remains unclear. The present study was carried out to examine the effect of low, high fat or high fat high fructose feeding on lipid metabolism, the antioxidant status in mice and the correlations between obesity, type2 diabetes, oxidative stress and NLRP3 in all groups

\section{Material AND Methods}

30 male albino mice weighting 30-40g were obtained from the animal house of National Research Center (NRC), Giza, Egypt. The animals were housed in individual suspended stainless steel cages in controlled environment (22-25oC) and 12hours light, 12hoursdark with food and water freely available. Low fat diet (LF) was prepared according to Reeves [15], HF according to Buettner et al. [16] and HFF according to Christopher et al. [17]. The mice were divided to the following groups 10 mice per each: Group1: mice fed $15 \mathrm{~g}$ /day from LF.Group2: fed $15 \mathrm{~g}$ /day from HF. Group3:fed $15 \mathrm{~g}-$ /day from HFF. At the start of experiment all groups fed LF diet at incubation period for 2 weeks. Weight determined at zero time and end point after 2 months. After (10 weeks), animals were kept fasting for 12 hours before blood sampling. Blood was withdrawn from the retro-orbital venous plexus of the eye using a capillary tube, under light anesthesia by diethyl ether. Blood was collected in tubes for the estimation of lipid parameters, glucose by enzymatic colorimetric method using Spectrum kit, insulin level was determined using INS-ELISA Kit BioSoure, Belgium) and NLRP3 supplied from NOVA Company for detection of nephropathy. Kidneys were removed quickly on ice and homogenized with phosphate buffer PH 7.4 for estimation of oxidative stress levels in kidney tissues.Insulin resistance was calculated from the equation:

Insulin resistance $=$ Fasting glucose $(\mathrm{mg} / \mathrm{dl}) \times$ fasting insulin $/ 22.5(\mu \mathrm{IU} / \mathrm{ml})$

\subsection{Statistical Methods}

The collected data were coded, tabulated, and statistically analyzed using IBM SPSS statistics (Statistical Package for Social Sciences) software version 22.0, IBM Corp., Chicago, USA, 2013. Descriptive statistics were done for quantitative data as minimum\& maximum of the range as well as mean \pm SD (standard deviation) for quantitative parametric data. Inferential analyses were done for quantitative variables using paired t-test in cases of two dependent groups with parametric data, ANOVA Test for more than two independent groups with post hoc Tukey HSD test. While correlations were done using Pearson correlation for numerical parametric data. The level of significance was taken at $\mathrm{P}$ value $<0.050$ is significant, otherwise is non-significant.

\section{RESULTS}

Figure 1show: no significant difference between study groups regarding weight before intervention. Weight after intervention was highest in HFF followed by HF and lowest in LF group, all differences between groups were significant. 


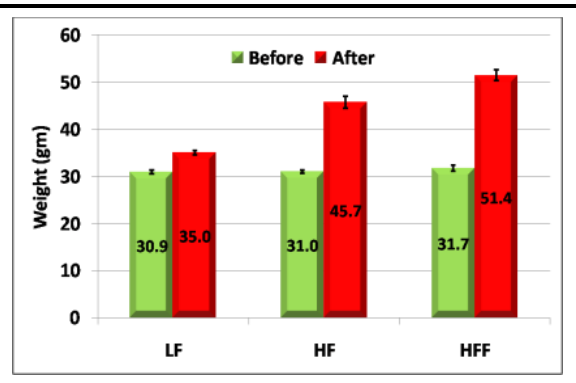

Figure1. weight of mice before and after intervention

Figure (2, 3 and 4$)$ shows a significant increase $(\mathrm{P} \leq 0.001)$ in serum glucose, insulin and HOMA-IR after feeding HFF and HF groups compared to LF group. Also, glucose level, insulin resistance in HFF group increase significantly compared to HF group.

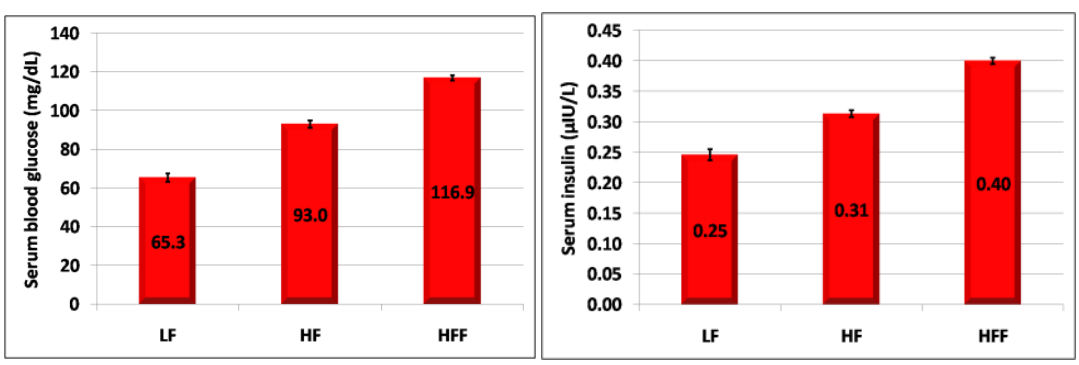

Figure2. serum glucose after intervention

Figure3. Serum insulin after intervention

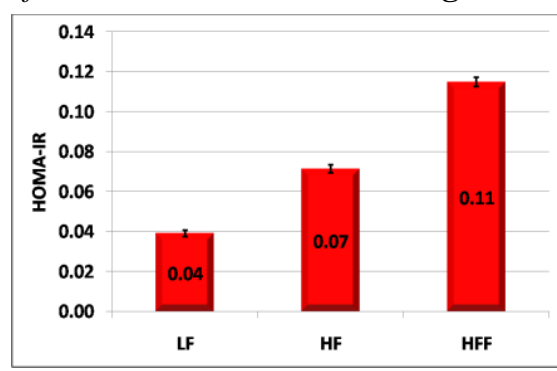

Figure4. HOMA-IR after intervention

Figures $(5,6$ and 7$)$ show a significant increase $(\mathrm{P} \leq 0.001)$ in serum total cholesterol, triacylglycerol and serum HDL-C after feeding HFF and HF groups compared to LF group. Also, cholesterol level and triacylglycerol in HFF group were increased significantly compared to HF.

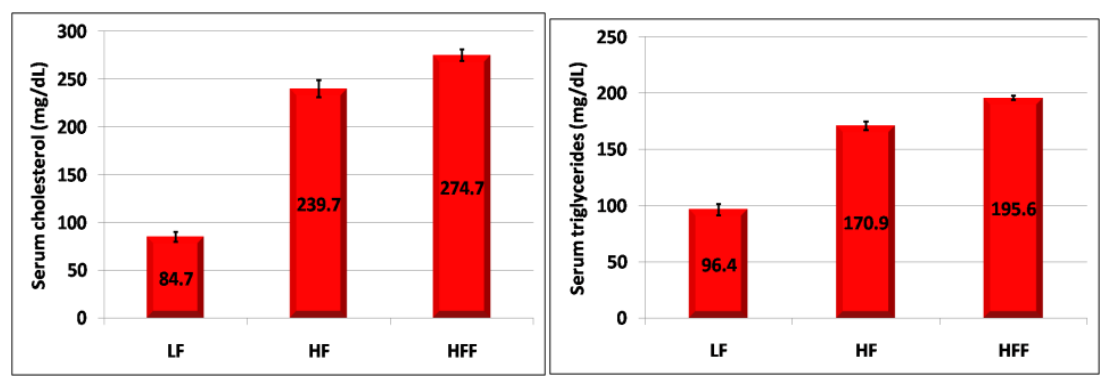

Figure5. Serum total cholesterol after intervention

Figure6. Serum triacylglycerols after intervention

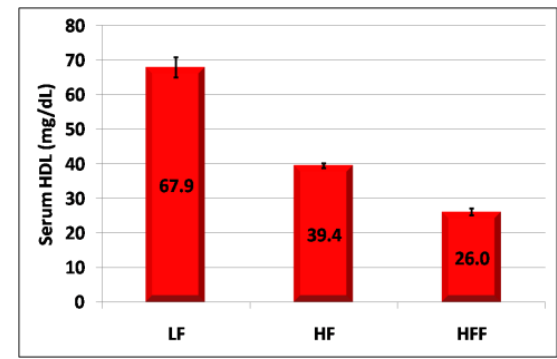

Figure7. Serum HDL-C after intervention

NO and MDA were significantly increased $(\mathrm{P} \leq 0.001)$ in kidney after feeding HFF and HF groups compared to LF group as shown in figures (8 and 9 ) and in HFF group compared to HF group. 


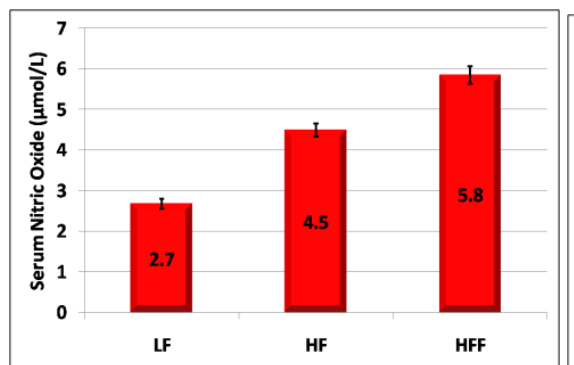

Figure8. Serum Nitric Oxide after intervention

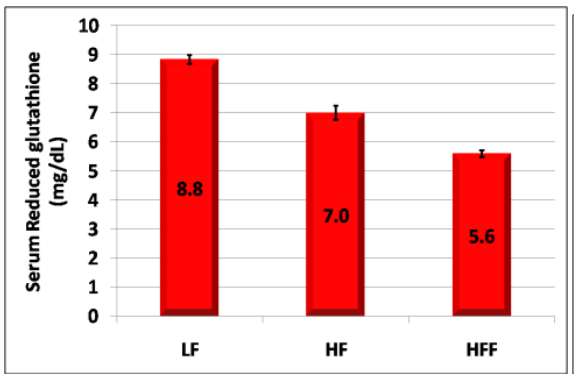

Figure10. kidney reduced glutathione after intervention

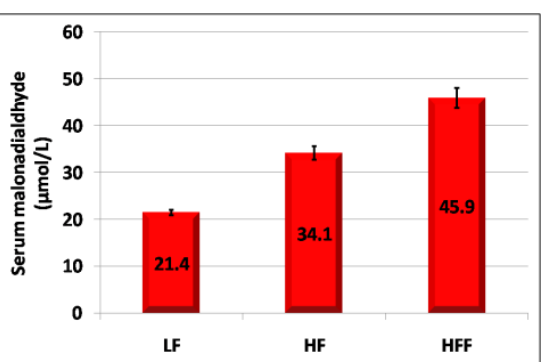

Figure9. kidney Malonadialdhyde after intervention

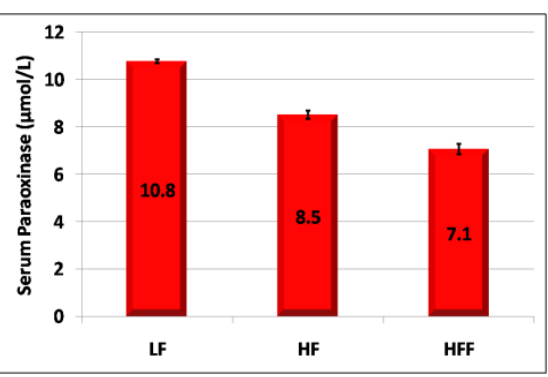

Figure11. kidney Paraoxinase lafter intervention

But GSH and PON1 were significantly decreased (P $\leq$ 0.001) in kidney after feeding HFF and HF groups compared to LF group as shown in figures (10 and 11)and in HFF group compared to HF group

Figure (12) show a significant increase $(\mathrm{P} \leq 0.001)$ in Serum NLPR3 after feeding HFF and HF groups compared to LF group. Also, serum NLPR3 level in HFF group was increased significantly compared to HF group.

There were significant positive correlations between NLPR3 and weight, glucose, insulin, HOMA IR, cholesterol, triacylglycerols, NO and MDA. But there were significant negative correlations between NLPR3 and HDL, GSH and PON1as shown in tables (1 and 2).

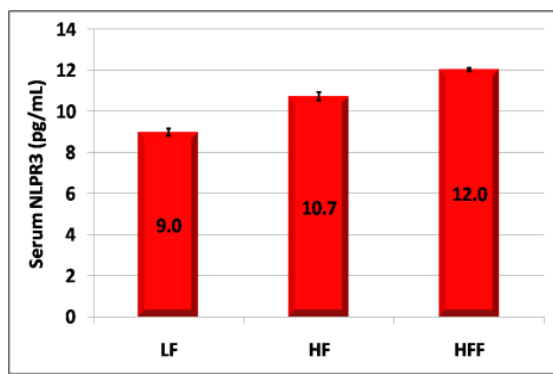

Figure2. Serum NLPR3 after intervention

Table1. Correlation between NLPR3 and other parameters

\begin{tabular}{|c|c|c|c|c|}
\hline & & LF & HF & HFF \\
\hline \multirow{2}{*}{ Weight } & $\mathbf{r}$ & 0.871 & 0.860 & 0.807 \\
\hline & p & 0.011* & $\mathbf{0 . 0 1 3}^{*}$ & 0.028* \\
\hline \multirow{2}{*}{ Glucose } & $\mathbf{r}$ & 0.709 & 0.876 & 0.727 \\
\hline & p & 0.047* & 0.010* & 0.044* \\
\hline \multirow{2}{*}{ Insulin } & $\mathbf{r}$ & 0.880 & 0.952 & 0.909 \\
\hline & p & 0.009* & 0.001* & 0.005* \\
\hline \multirow{2}{*}{ HOMA-IR } & $\mathbf{r}$ & 0.896 & 0.988 & 0.696 \\
\hline & p & 0.006* & $<0.001 *$ & $0.042 *$ \\
\hline \multirow{2}{*}{ Cholesterol } & $\mathbf{r}$ & 0.800 & 0.915 & 0.885 \\
\hline & $\mathbf{p}$ & 0.031* & 0.004* & 0.008* \\
\hline \multirow{2}{*}{ Triacylglycerols } & $\mathbf{r}$ & 0.709 & 0.903 & 0.703 \\
\hline & p & 0.047 & $0.005^{*}$ & 0.048* \\
\hline \multirow{2}{*}{ HDL } & $\mathbf{r}$ & -0.900 & -0.965 & -0.908 \\
\hline & $\mathbf{p}$ & 0.006* & $<0.001 *$ & $\mathrm{0.005}^{*}$ \\
\hline
\end{tabular}

Pearson's correlation test, *Significant 
Effect of Western Style Diet on NLRP3 Inflammasome and its Correlation with Metabolic Syndromes

Table2. Correlation between NLPR3 and other parameters after intervention in study groups

\begin{tabular}{|c|c|c|c|c|}
\hline & & LF & HF & HFF \\
\hline \multirow{2}{*}{ NO } & $\mathbf{r}$ & 0.990 & 0.964 & 0.961 \\
\cline { 2 - 5 } & $\mathbf{p}$ & $\mathbf{0 . 0 0 1} *$ & $<\mathbf{0 . 0 0 1} *$ & $\mathbf{0 . 0 0 1}$ \\
\hline \multirow{2}{*}{ Malon } & $\mathbf{r}$ & 0.704 & 0.993 & 0.912 \\
\cline { 2 - 5 } & $\mathbf{p}$ & $\mathbf{0 . 0 4 7}$ & $<\mathbf{0 . 0 0 1} *$ & $\mathbf{0 . 0 0 4}$ \\
\hline \multirow{2}{*}{ Red Glut } & $\mathbf{r}$ & -0.653 & -0.913 & -0.916 \\
\cline { 2 - 5 } & $\mathbf{p}$ & $\mathbf{0 . 0 1 2}$ & $\mathbf{0 . 0 0 4}$ & $\mathbf{0 . 0 0 4}$ \\
\hline \multirow{2}{*}{ Para } & $\mathbf{r}$ & -0.792 & -0.938 & -0.963 \\
\cline { 2 - 5 } & $\mathbf{p}$ & $\mathbf{0 . 0 3 4}$ & $\mathbf{0 . 0 0 2}$ & $<\mathbf{0 0 1}$ \\
\hline
\end{tabular}

Pearson's correlation test, *Significant

\section{DisCUSSION}

The present experiment elucidates that exposure to a HF and HFF diets led to increases in weight gain and impairments in blood glucose regulation. The body weight was increased significantly in HFF group than HF group. The results of the present study are in agreement with previous studies, especially which involving hamsters, which have shown that feeding high-fat diets and high-fructose diets led to rapid weight gain [18].This explained by the fact that obesity is a medical condition in which increase body fat has accumulated to the extent that it may unfavorable on health [19]. The present effects of the high-fat diet are in accordance with previous experiments by Petro et al. [20] that found that animals fed a high-fat diet had significantly higher blood glucose levels than that fed a low-fat diet, which led to the conclusion that high-fat diets negatively affect blood glucose levels in animals. Results of the present study revealed that HF and HFF groups recorded significant increases in serum insulin, plasma glucose and HOMA IR compared to control. Also serum insulin, plasma glucose and HOMA IR were significantly increased in HFF than HF. Parallel results were recorded by Bergmann and Syniewska [21] who found a significant increase of insulin levels could be referred to the induction of peripheral resistance with abdominal obesity, due to secretion of inflammatory factors and specific adipocytokines. The increased release of fatty acids, pro inflammatory cytokines, glycerol and other factors resulted in the development of insulin resistance. Elevated plasma levels of FFA are known to cause peripheral (muscle) insulin resistance by inhibiting insulin stimulated glucose up take and synthesis of glycogen. The mechanism involves intra myocellular accumulation of diacylglycerol and activation of protein kinase C; in addition, FFAs also cause hepatic insulin resistance by inhibiting insulin mediated suppression of glycogenolysis [22].

Results of the present study revealed that HF and HFF groups showed a significant increase in serum triacylglycerol and total cholesterol while HDL-C concentration was significantly decreased as compared to control. Also triacylglycerol and total cholesterol were significantly increased in HFF than HF. Also these results are in agreement with Woodie and Blythe [23].The increased cholesterol in HFF and HF groups may be due to the increased metabolism of glucose to pyruvate in liver. Then the pyruvate enters the liver mitochondria and converted to acetylecoA which is the starting molecule for cholesterol synthesis. Piia [24] found that triacylglycerol is exchanged for cholesterol esters by activity of cholesteryl ester transfer protein. This process results in triacylglycerol enriched HDL-C which catabolized more rapidly [25]. The AMPK (AMP-activated protein kinase) is an intracellular energy and nutrient sensor [26], sterol regulatory element binding protein (SREBP) is a key regulator of intracellular lipid metabolism that it considered the substrate of AMPK, where mRNA of hepatic SREBP-2 and SREBP-1c are linked with AMPK inhibition in obesity-induced insulin-resistant, that correlates with an increase in hepatic expression of SREBP-2 and SREBP-1c and its target genes [27]. Consistently, mRNA expression of SREBP-1c target genes including acetyl-CoA carboxylase (ACC1), fatty acid synthase (FAS), and stearoyl-CoA desaturase-1 (SCD1) is stimulated, suggesting increases of rates of fatty acid and triacylglycerol synthesis in the liver. We have also demonstrated that the increased ROS production and reduced GSH concentration in kidney cells in groups was fed $\mathrm{HF}$ and HFF, has resulted in compromised mitochondrial bioenergetics, resulted in inhibition of the activities of the respiratory complexes followed by a reduction in ATP synthesis finally lead to increased mitochondrial dysfunction [28].In the current study there were significant decreases in kidney PON1and GSH level and a significant increase in kidney MDA and NO in HFF and HF compared to LF. These results were in agreement with Lozano et al. [29] who indicated that generation of free radicals promotes lipid peroxidation in HF and HFF groups. In agreement with 
Oraby et al. [30] who indicated that serum NO was significantly increased in HFF and HF rats compared to control. This result may be due to the fact that increased oxidative stress and subsequent activation of the transcription factor NF-B have been associated with the development of late diabetic complications; NF-B enhances nitric oxide production, which is supposed to be a mediator of islet beta-cell damage [31].Increased generation of nitric oxide can be harmful to kidney functions, where synthesis of nitric oxide by both the inducible and constitutive nitric oxide synthase isoforms participates to the activation of apoptotic pathways in the kidney. NO itself is a free radical and can react with many other free radicals e.g., superoxide radical, generating peroxynitrite radical, capable of resulting oxidative changes to macromolecules [32]. In our study we found that the mean value level of NLRP3 HF and HFF were significantly increased compared to low fat group and this in agreement with [33].

The NLRP3 inflammasome is a key mediator of metabolic inflammation and disorder in ATM saturated fatty acids inhibit regulation of energy storage by lowering AMP kinase activity lead to releasing of ROS and production of IL1-1 $\beta$ which made a series of metabolic disorders as it promotes insulin resistance through serine phosphorylation of insulin receptor substrate-1(IRS-1) that impairs engagement of the insulin receptor (IR) with IRS-1. It also enhances a direct-insulin resistance by promoting expression of tumor necrosis factor $\alpha$ (TNF- $\alpha$ )[34].Dysfunctional of adipose tissue in lipid metabolism is due to increases intriacylglycerol and cholesterol leads to increased circulating free fatty resulting in obesity. This obesity with chronic inflammation initiates a state of insulin resistance. The secretion of chemoattractants; such as MIF andMCP-1, cytokines IL-1 $\beta$, TNF- $\alpha$ and IL-6are associated with inflammation which isa critical mediator in obesity-induced insulin resistance [35].Our study shows a positive correlation between total cholesterol and triacylglycerol withNLRP3 which suggested that activation of NLRP3 inflammasome lead to produce IL1-1 $\beta$ which made a series of metabolic disorder. Also there is a negative correlation between HDL-C, PON1 and GSH withNLRP3 which suggested that NLRP3metabolic inflammation and disorder in ATM saturated fatty acids inhibit regulation of energy storage.

\section{Conclusion}

We concluded that the inflammasome NLRP3 is an important marker that correlates between obesity, type 2 diabetes, oxidative stress and inflammation, so it is recommended to depend on as metabolic syndrome marker. Also we recommended further studies to inactivate the enzymes responsible for the formation of NLRP3 to decrease the resulted metabolic syndromes

\section{REFERENCES}

[1] Oleg, V. (2016): Western-style diet, sex steroidandmetabolism, Biochim. Biophys. Acta

[2] Covas M.I. (2007): Olive oil and the cardiovascular system.Pharmacol Res55:175-186.

[3] Sofi F., Cesari F., Abbate R., Gensini G.F., Casini A.(2008): Adherence toMediterranean diet and health status: meta-analysis (Abstract). BMJ 337: a1344.

[4] NCD Risk factor Collaboration (2016): Trends in adult body-mass index in 200 countries from 1975 to 2014: a pooled analysis of 1698 population- based measurement studies with 19.2 million participants. Lancet 387: 1377-1396.

[5] Heidemann C., Schulze M.B., Franco O.H., van Dam R.M., Mantzoros C.S., Hu F.B., (2008): Dietary patterns and risk of mortality from cardiovascular disease, cancer, and all causes in a prospective cohort of women. Circulation 118: 230 -237.

[6] Odermatt A., (2011):The Western-style diet: a major risk factor for impaired kidney function and chronic kidney disease. Am J Physiol Renal Physiol; 301: F919-F931.

[7] Cohen J.C., Horton J.D., Hobbs H.H. (2011): Human fatty liver disease: old questions and new insights. Science 332: 1519-1523.

[8] Prentice A., (2004): Diet, nutrition and the prevention of osteoporosis.Public Health Nutr 7: 227243.

[9] Jurgens H., Haass W., Castaneda T.R., Schurmann A., Koebnick C., Dombrowski F., Otto B., Nawrocki A.R., Scherer P.E., Spranger J., Ristow M., Joost H.G., Havel P.J., Tschop M.H. (2005): Consuming fructosesweetenedbeverages increases body adiposity in mice. Obes Res 13: 1146-1156. 
[10] Stanhope K.L., Schwarz J.M., Keim N.L., Griffen S.C., Bremer A.A., Graham J.L., Hatcher B., Cox C.L., Dyachenko A., Zhang W., McGahan J.P., Seibert A., Krauss R.M., Chiu S., Schaefer E.J., Ai M., Otokozawa S., Nakajima K., Nakano T., Beysen C., Hellerstein M.K., Berglund L., Havel P.J. (2009): Consuming fructose-sweetened, not glucose-sweetened, beverages increases visceral adiposity and lipids and decreases insulin sensitivity in overweight/obese humans. J Clin Invest 119: 1322-1334.

[11] World Health Organization, (2011): Global Status Report on Non communicable Diseases 2010, World Health Organization, Geneva, Switzerland.

[12] American Diabetes Association(2017):Diagnosis and classification of diabetes mellitus. Diabetes Care.37.

[13] Stienstra R, van Diepen J.A., Tack C.J., et al (2011): Inflammasome is a central player in the induction of obesity and insulin resistance. ProcNatlAcadSci USA 2011; 108: 15324-15329.

[14] Gersch M.S., Mu W., Cirillo P., et al. (2007): Fructose, but not dextrose, accelerates the progression of chronic kidney disease. Am J Physiol Renal Physiol; 293: F1256-F1261.

[15] Reeves P.G. (1997): Components of the AIN-93 diets as improvements in the AIN-76A diet.J Nutr. 27(5 Suppl):838S-841S.

[16] Buettner R., Scholmerich J. and Bollheimer L.C. (2007): High-fat diets: modeling the metabolic disorders of human obesity in rodents. Obesity (Silver Spring) 15: 798-808.

[17] Christopher R., WILSON., Mai K., TRAN., Katrina L., SALAZAR., Martin E., YOUNG. and Heinrich. (2007) :TAEGTMEYER Western diet, but not high fat diet, causes derangements of fatty acid metabolism and contractile dysfunction in the heart of Wistar rats Biochem. J. 406 (457-467).

[18] Alsaif M.A. and Duwaihy M.M.S. (2004): Influence of dietary fat quantity and compositionon glucose tolerance and insulin sensitivity in rats. NutrRes. ;24:417-25.

[19] RobertI., Swamy L., and Constantinos S, P.(2007): Emerging mobile health systems . Medical ., 623 pages.

[20] Petro, A.E.; Cotter, J.; Cooper, D.A.; Peters, J.C. and Surwit, R.S.(2004):Fat,carbohydrate, and calories in the development of diabetes and obesity inthe C57BL/6J mouse. Metabolism, 53:4547.

[21] Bergmann K. and Sypniewska G. (2013): Diabetes as a complication of adipose tissue dysfunction. Is there arole for potential new biomarkers? Clin. Chem. Lab. Med. jan ; 51(1) : $177-85$.

[22] Boden G. (2003): Effect of free fatty acids on glucose metabolism : significance for insulin resistance and type 2 diabetes. Exp. Clin. Endocrinol diabetes. 111(3): 121-4.

[23] Woodie L., Blythe S.(2017): The differential effects of high-fat and high-fructose diets on physiology and behavior in male rats.NutrNeurosci. Feb 14:1-9.

[24] Piia P.S., Helena K.G., and Tatu A.M. (2002): Diabetes contributes to cholesterol metabolism regardless of obesity. Diabetes care, 25(9):1511-1515.

[25] Valkenburn O., Regine P.M., Theuissens H.P., Smedts M. and Gessje M. (2008): A more atherogenicserum lipoprotein profile is present in woman with Pcos. J.clinical. endocrinal .meta., vol 93: 470-476.

[26] Hardie D.G., Ross F.A., Hawley S.A. (2012): AMPK: a nutrient and energy sensor that maintains energy homeostasis. Nat Rev Mol Cell Biol 13(4):251-262.

[27] Li L., Astor B.C., Lewis J., Hu B., Appel L.J., Lipkowitz M.S., Toto R.D.,Wang X., Wright J.T. J.r., Greene T.H. (2011): Longitudinal progressiontrajectory of GFR among patients with CKD. Am J Kidney Dis 59:504-512.

[28] Raza H., Subbuswamy K.P., John A., Avadhani N.G. (2011): Impaired mitochondrial respiratory functions and oxidative stress in streptozotocin-induced diabetic rats. Int. J. Mol. Sci, 12, 31333147.

[29] Lozano-Baena M.D., Tasset I., Muñoz-Serrano A., Alonso-Moraga Á., de Haro-Bailón A. (2016): Cancer Prevention and Health Benefices of Traditionally Consumed Boragoofficinalis Plants. Nutrients.;8:48. 
Fatma Farag Abdel Hamid et al.

[30] Oraby H.A., Hassan A.A., AbouMossallam A.A. (2008): Screening food products for the presence of CaMV 35S promoter and NOS3' terminator. J. Sci. Food Agric. 85:1974-1980.

[31] Maritim A.C., Sanders R.A., Watkins J.B., (2003): Diabetes, oxidative stress and antioxidants: a review, Journal of Biochemical and Molecular Toxicology; 17: 24-38.

[32] Abdel-Salam, O.M.E., Salem N. and Hussein J.S. (2011): Effect of aspartame on oxidative stress and monoamine neurotransmitter levels in lipopolysaccharide-treated mice. Neurotox Res., 21(3):245-255.

[33] Yan Y. et al. (2015): Dopamine controls systemic inflammation through inhibition of NLRP3 inflammasome. Cell 160, 62-73.

[34] Vandanmagsar B., Youm Y-H.H., Ravussin A., et al. (2011): The NLRP3 inflammasome instigates obesity-induced inflammation and insulin resistance. Nat Med; 17: 179-188.

[35] Nishio M., Yoneshiro T., Nakahara M., Suzuki S., Saeki K. K., Hasegawa M., et al. (2012): Production of functional classical brown adipocytes from human pluripotent stem cells using specific hemopoietin cocktail without gene transfer. CellMetab. 16, 394-406.

Citation: F. Abdel Hamid, "Effect of Western Style Diet on NLRP3 Inflammasome and its Correlation with Metabolic Syndromes", International Journal of Clinical Chemistry and Laboratory Medicine (IJCCLM), vol. 3, no. 2, p. 8, 2017. http://dx.doi.org/10.20431/2455-7153.0302001

Copyright: () 2017 Authors. This is an open-access article distributed under the terms of the Creative Commons Attribution License, which permits unrestricted use, distribution, and reproduction in any medium, provided the original author and source are credited. 